\title{
The use of the T-history method to estimate thermal capacity and latent heat for RT15 and RT18 materials
}

\author{
Paulina Kapica ${ }^{1, *}$, Jarosław Karwacki ${ }^{1}$, and Sebastian Bykuć ${ }^{1}$ \\ ${ }^{1}$ The Szewalski Institute Of Fluid Machinery, Polish Academy of Sciences, Fiszera 14, 80-231 Gdańsk, Poland
}

\begin{abstract}
The paper presents theoretical assumptions and implementation of the T-history method, which was used to estimate the thermal capacity and latent heat for phase change materials (PCMs). The research equipment, measurement system and obtained results were discussed. The T-history method was used to investigate the heating and cooling process of two PCMs, sold under the trade names of RT15 and RT18. Obtained results were compared with the manufacturer's data. The analysis of the research results has led to the conclusion that the presented measurement method made it possible to perform a preliminary verification of the enthalpy-temperature dependency of the PCMs. The method can be also used for studying different commercial materials and estimating this dependency for materials of yet unknown properties.
\end{abstract}

\section{Introduction}

The knowledge of thermophysical properties of heat storage materials is essential when designing heat and cold storage systems. In the case of solids in which only sensible heat is used, it is sufficient to know their specific heat and thermal conductivity. When liquids are used for heat storage purposes, the process of convective heat transfer should also be taken into account in the majority of design solutions. A much more difficult task is to design a heat store in which a PCM (phase change material) is employed. In such a case, it is necessary to know the specific heat for both the liquid and solid states ( $c_{p, s}$ and $c_{p, l}$ respectively) as well as the specific heat of fusion, which is related to the solid-liquid phase change. In most cases, these parameters can be determined using the well-known differential scanning calorimetry (DSC) technique. However, this method requires expensive laboratory equipment. Another (much simpler) solution is to use the T-history method, which was presented for the first time in publication [1]. This method has been developed and improved over many years [2]. Due to the possibility of using samples of PCMs with masses greater than $50 \mathrm{mg}$ in the tests, the obtained results may be closer to reality.

In this paper, the T-history method was used to investigate specific heat and specific heat of fusion of two PCMs, sold under the trade names of RT15 and RT18. Fig. 1 and Fig. 2 presents the partial enthalpy distribution for those materials, that is the amount of heat /cold from unit of mass corresponding to a given temperature.The presented research had the following objectives:

- developing the test rig and measurement procedures for the implementation of the T-history method,
- validating the measurement method based on data given for materials sold on the market,

- determining further actions that can be taken in order to improve the reliability of the obtained results.

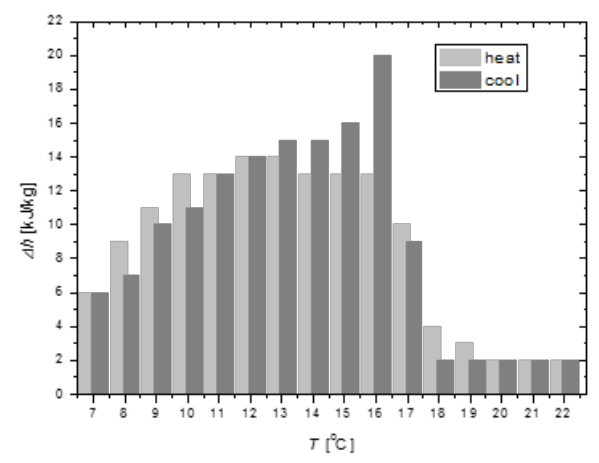

Fig. 1. Partial enthalpy distribution of phase change material RT15.

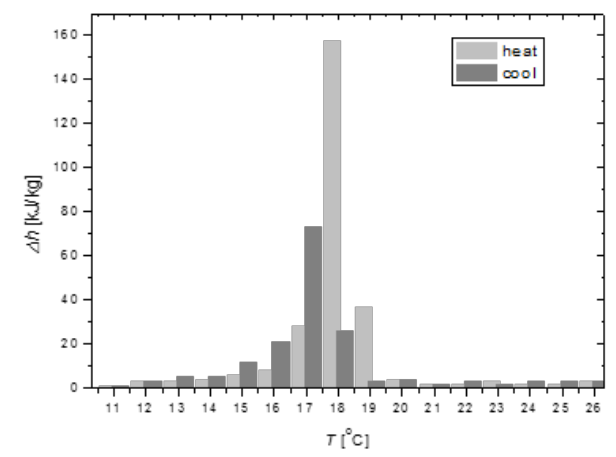

Fig. 2. Partial enthalpy distribution of phase change material RT18.

\footnotetext{
Corresponding author: paulina.kapica@imp.gda.pl
} 


\section{Calculation method}

The T-history method is a thermal analysis method that is based on registering temperatures of the investigated materials within a certain time period. Material samples of the proper diameter and length should be used. It is necessary that at least one of the materials to be tested has well-known thermal properties. Such a material is called "reference material". In the temperature range of 0 ${ }^{\circ} \mathrm{C}-100{ }^{\circ} \mathrm{C}$, water is usually used as a reference material. A comparison with the reference material allows to determine the phase transition temperature and then the thermal capacity as well as the latent heat of PCMs.

When using the above-mentioned method, the test tube with a material to be tested should be immersed in a liquid whose temperature significantly differs from the phase transition temperature. After the thermal equilibrium is reached, the tested materials are usually immediately exposed to ambient temperature. Temperature is measured using sensors inserted directly into material samples. In the T-history method, material samples are heated after their placement in the so-called "water bath", as shown in Fig. 3.

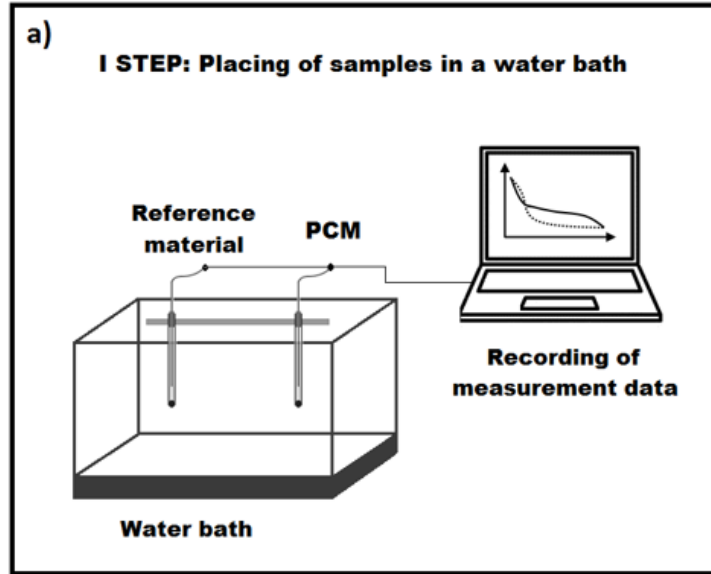

b)

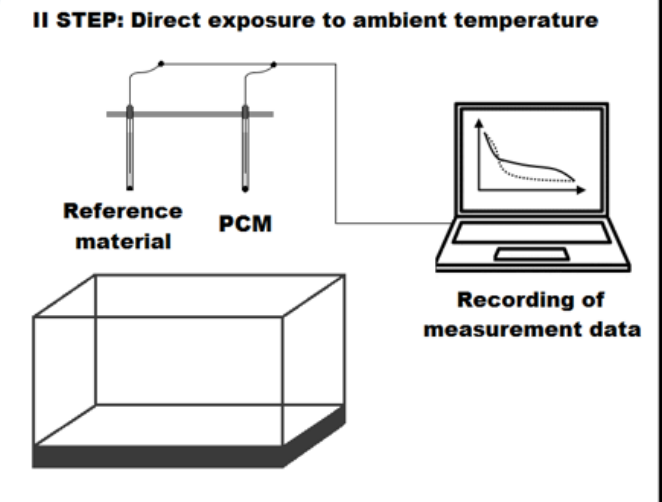

Fig. 3. T-history method - schematic diagram of experimental apparatus.

A prerequisite for the use of the T-history method is to make ensure that the material to be tested will have even temperature distribution along its entire crosssection. This prerequisite is met if the Biot number is lesser than 0.1 . This is done by choosing appropriate dimensions of test tubes and paying a close attention to the adequate conduct of the experiment.

The first step of the T-history method is to place test tubes inside the hot water tank (Fig. 3a). Afterwards, they are heated until the temperature reaches $T_{0}$, namely the temperature that is greater than the melting point of the PCM $\left(T_{1}\right)$. The test tubes are removed from the tank as soon as a steady state is reached. After the removal, they are kept at the ambient temperature $\left(T_{a}\right)$, which usually is the temperature of the ambient air. In the configuration described herein, the tested materials were taken out from the water bath at the appropriate moment (Fig. 3b). It is important that the ambient temperature $\left(T_{a}\right)$ should be significantly lower than the freezing point of the PCM $\left(T_{s}\right)$. As soon as the heated test tubes (with the PCM and the reference material) are exposed to ambient temperature the cooling process commences. The temperature of both materials was measured, which made it possible to plot the graphs presenting their temperature as functions of time. The measurement started at a temperature of $T_{0}$ and ended after a temperature of $T_{e}$ was reached. The temperature denoted by $T_{e}$ has to be lower than the temperature denoted by $T_{s}$ (solidification point of the PCM). As a result, temperature profiles of the PCM and the reference material ( $T_{m}$ and $T_{r}$ respectively) are obtained.

On the graph that shows the cooling process curves one can differentiate the three following temperature ranges:

1 - from $T_{0}$ (start of the measurement) to $T_{1}$ that is the melting point of the PCM (in this temperature range the PCM is in the liquid state),

2 - from $T_{1}$ to $T_{s}$ that is the solidification point of the PCM (the range in which the phase change takes place),

3 - from Ts to Te which is the temperature registered at the end of the experiment (in this temperature range the PCM is in the solid state).

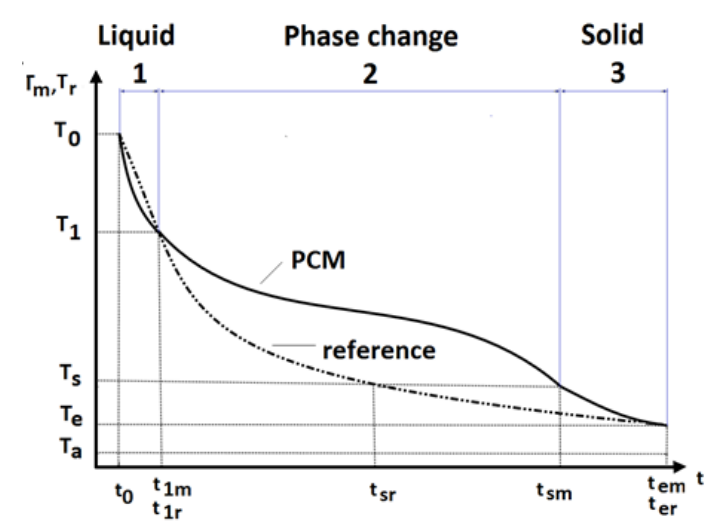

Fig.4. Cooling process curves for PCM and reference material with division into PCM phase zones.

In the first zone marked in Fig. 4, the heat transfer in both test tubes is defined by the following equations [3]:

$$
\begin{gathered}
\left(m_{t m} c_{p t m}+m_{m} c_{p m l}\right)\left(T_{0}-T_{1}\right)=h_{1} S_{t m} A_{1 m} \\
\left(m_{t r} c_{p t r}+m_{r} c_{p r}\right)\left(T_{0}-T_{1}\right)=h_{1} S_{t r} A_{1 r}
\end{gathered}
$$


where:

$m_{m}, m_{r}-$ mass of the PCM / reference material

$m_{t m}, m_{t r}-$ mass of the test tube in which the PCM / reference material was inserted

$c_{p m l} c_{p r}-$ specific heat of the PCM in the liquid state / reference material

$c_{p t}-$ specific heat of the test tube

$h$-convective heat-transfer coefficient

$S_{t}$ - convective heat transfer area

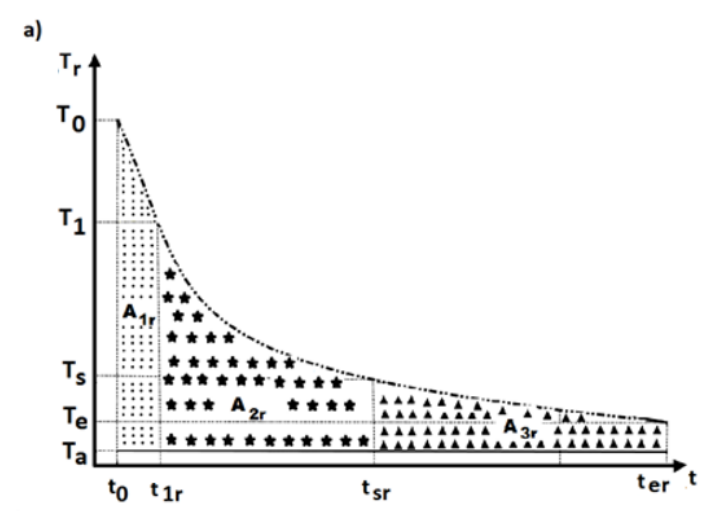

b)

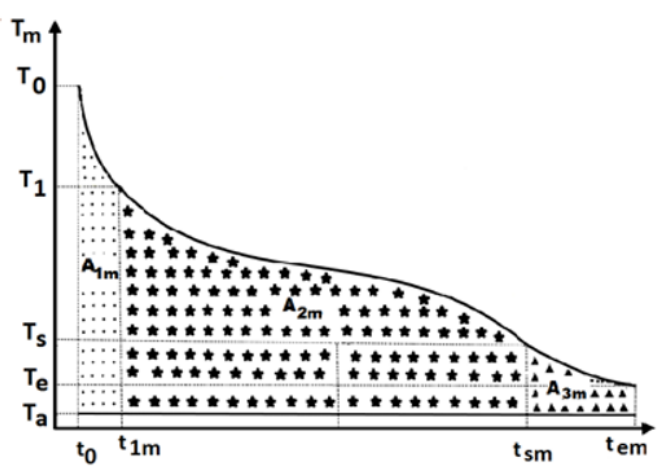

Fig.5. Thermal capacity of the reference material (a) and PCM (b) at temperature intervals.

The next zone is the PCM phase change zone and the heat transfer can be expressed by equations (3) and (4).

$$
\begin{gathered}
m_{t m} c_{p t m}\left(T_{1}-T_{s}\right)+m_{m} H_{m}=h_{2} S_{t m} A_{2 m} \\
\left(m_{t r} c_{p t r}+m_{r} c_{p r}\right)\left(T_{1}-T_{s}\right)=h_{2} S_{t r} A_{2 r}
\end{gathered}
$$

The last zone corresponds to the temperature range in which the PCM occurs in the solid state. The heat transfer balance between both materials (PCM and reference material) and their surroundings is given by equations (5) and (6).

$$
\begin{gathered}
\left(m_{t m} c_{p t m}+m_{m} c_{p m s}\right)\left(T_{s}-T_{e}\right)=h_{3} S_{t m} A_{3 m} \\
\left(m_{t r} c_{p t r}+m_{r} c_{p r}\right)\left(T_{s}-T_{e}\right)=h_{3} S_{t r} A_{3 r}
\end{gathered}
$$

For the purposes of the above equations, the magnitude of the areas shown in Fig. 5 can be calculated using the following integrals:

$$
\begin{gathered}
A_{1 r}=\int_{t_{0}}^{t_{1 r}}\left(T_{r}(t)-T_{a}\right) d t \\
A_{2 m}=\int_{t_{1 m}}^{t_{2 m}}\left(T_{m}(t)-T_{a}\right) d t \\
A_{2 r}=\int_{t_{0}}^{t_{s r}}\left(T_{r}(t)-T_{a}\right) d t \\
A_{3 m}=\int_{t_{s m}}^{t_{\text {em }}}\left(T_{m}(t)-T_{a}\right) d t \\
A_{3 r}=\int_{t_{s r}}^{t_{\text {Er }}}\left(T_{r}(t)-T_{a}\right) d t
\end{gathered}
$$

Phase transition temperature values of a tested PCM are determined on the basis of the derivative of the cooling process curve. On the graph presenting this derivative, one should be able to notice two temperature peaks that correspond to the start and end moments of the phase transition. The first peak $\left(T_{l}\right)$, which occurs in the temperature range from $t_{0}$ to $t_{l m}$, corresponds to the beginning of phase change. The second peak occurs in the range from $t_{l m}$ to $t_{e m}$ and denotes the temperature $\left(T_{s}\right)$ at which the phase change is already over. The temperature denoted by $T_{e}$ is the temperature at which the experiment ended. At this temperature, the value which was close to the ambient temperature, the thermal equilibrium of the tested materials was reached.

Taking into account the calculated magnitude of the areas (formulas 7-12), dimensions of the test tubes, particular masses as well as the specific heat of the reference material and of the material from which test tubes are made, one can calculate the values of the following parameters:

- specific heat of the PCM in the liquid state:

$$
c_{p, m l}=M \frac{A_{1 m}}{A_{1 r}}-N
$$

- specific heat of the PCM in the solid state:

$$
c_{p, m s}=M \frac{A_{3 m}}{A_{3 r}}-N
$$

- latent heat:

$$
H_{m}=\left(\frac{M A_{2 m}}{M A_{2 r}}-N\right)\left(T_{1}-T_{s}\right)
$$

where:

$$
\begin{gathered}
M=\left(m_{t r} c_{p, t r}+m_{r} c_{p, r}\right) \frac{s_{t m}}{m_{m} s_{t r}} \\
N=m_{t m} \frac{c_{p, t m}}{m_{m}}
\end{gathered}
$$

The above-described procedure concerns the classical implementation of the T-history method that was proposed by Z. Yinping in 1999 [1]. As far as the research described in the following part of this article is concerned, material samples were not preheated to a suitably high temperature. On the contrary, they were cooled in the container with ice water so that the PCMs were completely solidified. After removing the samples $A_{1}$ rom thet $e^{t}$ water, their temperatures were registered until the materials reached the ambient temperature. This procedure resulted from the fact that the temperature at 
which the phase transition was complete is lower than the room temperature. It should be noted that formulas (14) to (17) remain valid also in such a case.

\section{Description of the experimental set-up}

The test rig, on which the RT15 and RT18 materials were tested, consists of the following elements:

- six test tubes made of glass

- thermally insulated container with ice water

- five thermocouples (CZAKI TP232) with a diameter of $1 \mathrm{~mm}$

- measurement system.

Initially, during the research were used the following test tube types: three cylindrical glass test tubes with an internal diameter of $16 \mathrm{~mm}$ and a length of $190 \mathrm{~mm}$ and three test tubes with an external diameter of $15 \mathrm{~mm}$ and a length of $160 \mathrm{~mm}$. The first two of the smaller test tubes were filled with materials that were to be tested (i.e. RT15 and RT18) and the last one of them with water that served as a reference material. The internal and external diameters of the test tubes as well as masses of the used materials are given in Table 1 .

Table 1. Internal and external diameters of the test tubes and masses of materials inserted into tchem.

\begin{tabular}{|c|c|c|c|c|c|c|}
\hline $\mathbf{d}_{\text {in }}$ & $\mathbf{d}_{\text {ext }}$ & $\mathbf{m}_{\mathbf{t}}$ & $\mathbf{m}_{\mathbf{m}}$ & $\mathbf{m}_{\mathbf{r}}$ & $\mathbf{m}_{\mathbf{t m}}$ & $\mathbf{m}_{\text {tr }}$ \\
\hline $\mathrm{mm}$ & $\mathrm{mm}$ & $\mathrm{kg}$ & $\mathrm{kg}$ & $\mathrm{kg}$ & $\mathrm{kg}$ & $\mathrm{kg}$ \\
\hline 13 & 15 & 0.023 & 0.0092 & 0.0119 & 0.032 & 0.035 \\
\hline
\end{tabular}

A thermocouple was inserted into each test tube. All three test tubes were fixed to a triangular plate made of a polycarbonate, equipped with a metal handle. Since the test tubes were equidistant from each other, a uniform temperature distribution was obtained during measurements. The larger test tubes were attached to the lid of the thermally insulated container. That way, smaller test tubes can be easily inserted into those larger ones (Fig.6). The thermally insulated container was filled with ice water of a temperature of approx. $0{ }^{\circ} \mathrm{C}$. Polystyrene foam was used as an insulation material in the container.

The temperature measurement was conducted using three thermocouples inserted in test tubes with tested materials and one thermocouple placed inside the container with ice water. During the research, the ambient temperature was measured as well. The NI SCXI data acquisition and signal conditioning system, as well as the software created in the LABView environment, were employed for recording and archiving the measured temperatures.

After analysing the research results, it turned out that it was necessary to modify the test rig (Fig. 7a). Placing tested samples in the container with air and then inserting this container into the thermally isolated container with ice water allowed to cool the samples as a result of heat transfer. That way, the heat was freely exchanged between the warm samples and the cooled air surrounding them. It was decided to attach the container filled with air to the lid of the rectangular container, so that test samples could be placed therein (Fig. 7b). A special moisture absorbing material was put at the bottom of the smaller container. Other components of the test rig were left unchanged.

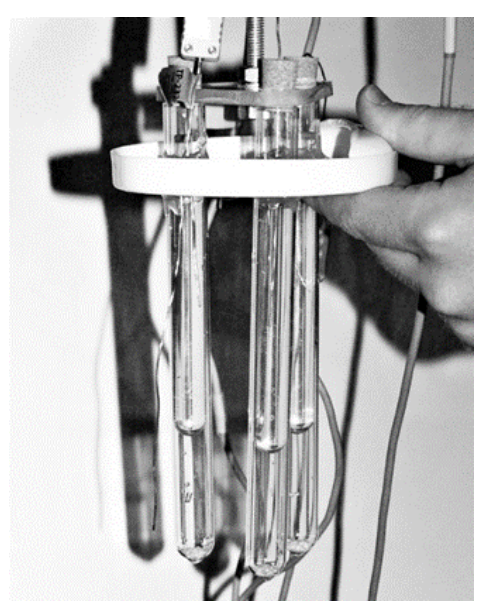

Fig.6. Placing smaller test tubes inside bigger ones that are attached to the lid of the container.

a)

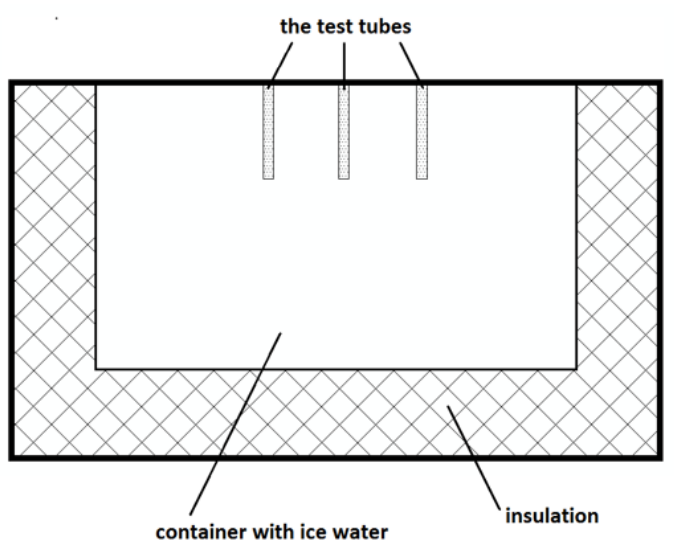

b)

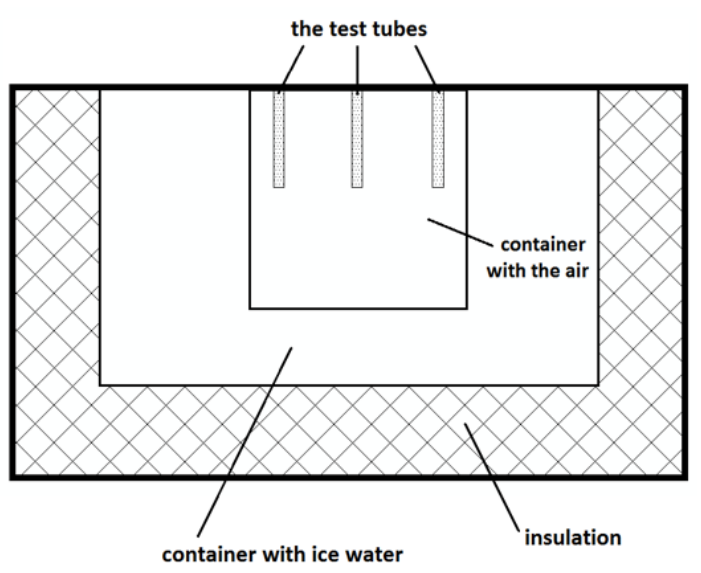

Fig.7. Cross-section of the test rig before (a) and after (b) its modification. 


\section{Methodology of tests}

The tests were started by filling smaller test tubes with water as well as RT15 and RT18 materials to the same volume $(12 \mathrm{ml})$. This was done at the ambient temperature conditions (at a temperature of $T_{a}$ ).

As mentioned, the way in which the research on RT15 and RT18 materials was carried out differs from the original $\mathrm{T}$-history method [1]. In the original $\mathrm{T}$ history method (and in its other implementations as well $[2,3,4,6])$, containers with materials are heated to a temperature much higher than the ambient temperature, and afterwards, they are abruptly exposed to the ambient temperature, causing them to cool down. To carry out a phase transition for some PCMs, a very wide temperature range is required. In practice, this involves using specialized equipment to achieve high temperatures.

In the experiment discussed herein, material samples are cooled by submerging them in ice water, in contrast to the usually performed heating up. At the beginning of the experiment, the tested materials had a temperature equal to the ambient temperature. The samples were placed in test tubes that were put in a thermally insulated container with ice water. The ice water had a lower temperature than the solidification point of the tested PCMs. About one hour later PCMs were completely solidified.

Then, after the temperature of the tested substances was stabilised, the samples were taken out of the insulated container and exposed to the ambient temperature. Heating started. During heating, the fusion process took place. The heat transfer between the samples and the surrounding air was not as intensive as during cooling because it lasted about four hours. During the heating process, a phase transition occurred since the ambient temperature was higher than the fusion temperatures of the PCMs.

As a result of the test, the full course of the phase transition was registered and temperature profiles over time were archived. The registration of measurement data was carried out in two stages. In the first stage, the temperature versus time was recorded during cooling of the samples in the ice water bath until a steady state was achieved. The second stage of recording started from the moment the samples were taken out from the ice water and ended after they reached stable temperatures equal to the ambient temperature. The results obtained from the measurement allowed to plot heating curves for RT15, RT18 and water.

In Fig. 9a, the heating curves concerning all materials (including water) abruptly changed their courses at a temperature of approx. $18{ }^{\circ} \mathrm{C}$. This is probably related to the formation of water droplets (as a result of condensation) on the walls of the test tubes just after removing them from the ice water bath and then their evaporation. That is the reason why an analysis of the properties of PCMs based on the heating process was abandoned.

The Biot number was only determined for the cooling process and its value was calculated according to the following formula:

$$
B i=\frac{\alpha \cdot \frac{V}{A}}{\lambda_{P C M}}
$$

Characteristic dimension in Biot number is equal to V/A (volume by surface area). The thermal conductivity coefficient $\left(\lambda_{\mathrm{PCM}}\right)$ was $0.2 \mathrm{~W} / \mathrm{m} \cdot \mathrm{K}$ (based on the manufacturer's data [8]). The convective heat-transfer coefficient $(\alpha)$ was calculated using the following formula [5]:

$$
\alpha=\frac{N u-\lambda}{l}
$$

where:

$$
\begin{gathered}
N u=0.54 \cdot(\mathrm{Gr} \times \mathrm{Pr})^{\frac{1}{4}} \\
G r \cdot \operatorname{Pr}=\frac{g \beta\left(T_{a}-T_{z}\right) l^{3}}{v^{2}} \times \operatorname{Pr} \\
\beta=\frac{1}{0.5\left(T_{z}+T_{a}\right)}
\end{gathered}
$$

The following parameters (at given temperatures) were read from thermodynamic tables contained in the REFPROP program: Prandtl numer (Pr), thermal conductivity coefficient $(\lambda)$ and coefficient of kinematic viscosity $(v)$. The Grasfoh number was calculated by knowing the ambient temperature $T_{a}$, the temperature inside the sample $T_{z}$, gravity $g$ and kinematic viscosity $v$. The mean value of the diameter of the samples (using in calculation) was $14 \mathrm{~mm}$. The Biot number was calculated for two values of the characteristic length $(l)$, namely $150 \mathrm{~mm}$ (corresponding to the length of the test tube) and $95 \mathrm{~mm}$ (corresponding to the height of the tested substance). Due to the lack of precision in the method of presenting the characteristic length $(l)$ in publications $[1,4]$, it was decided to present the Biot number for both values of $l$. The obtained results differ, which means that the value of the characteristic length has an impact on the value of the Biot number. Therefore, it is crucial to correctly interpret its value. Nevertheless, setting the value of the characteristic length (1) to the value equal to the height of the tested substance seems to be the most logical choice.

The equations presented above show that the Biot number is dependent (although not directly) on the obtained temperatures. In publications $[1,3]$, there is no information about the exact range of the Biot number during the carried out measurements. Their authors only ensured that the Biot number criterion was fulfilled $(\mathrm{Bi}<0.1)$.

In the conducted experiment, the calculated value of the Biot number for the initial test rig was higher by several orders of magnitude than the value given in the criterion and that was the case throughout the whole duration of the cooling process. Obtaining such a great value of the Biot number (in comparison with the value given in the criterion) was caused by the occurrence of the convective heat-transfer coefficient $(\alpha)$ in the range of $100-350 \mathrm{~W} / \mathrm{m}^{2} \cdot \mathrm{K}$ (depending on the temperatures obtained) for almost the entire duration of the test. This is the reason why the cooling process of samples was so 
intense. The obtained value of the Biot number during the cooling process for the initial test rig precluded the use of the T-history method and a modification of the test rig became necessary.

After upgrading the test rig, the material samples were tested once again. This time the samples were cooled down using cooled air. The ice water served for cooling the air surrounding the tested materials. After the cooling finished, the samples were heated in the same way as it had been done before the modification of the test rig.

The Biot number during the cooling process that took place on the modified test rig was calculated using formula (18) and its values for several selected temperatures are shown in Table 2.

Table 2. Biot number values for chosen temperatures after the modification of the test rig.

\begin{tabular}{|c|c|c|c|c|c|c|c|}
\hline $\mathbf{T}_{\mathbf{a}}$ & $\mathbf{T}_{\mathbf{z}}$ & $\mathbf{N u}_{\mathbf{1}}$ & $\mathbf{N u}_{\mathbf{2}}$ & $\boldsymbol{\alpha}_{\mathbf{1}}$ & $\boldsymbol{\alpha}_{\mathbf{2}}$ & $\mathbf{B i}_{\mathbf{1}}$ & $\mathbf{B i}_{\mathbf{2}}$ \\
\hline${ }^{\circ} \mathrm{C}$ & ${ }^{\circ} \mathrm{C}$ & {$[-]$} & {$[-]$} & $\mathrm{W} / \mathrm{m} 2 \mathrm{~K}$ & $\mathrm{~W} / \mathrm{m} 2 \mathrm{~K}$ & {$[-]$} & {$[-]$} \\
\hline 0.84 & 22.2 & 30.46 & 21.62 & 4.89 & 5.48 & 3.66 & 2.60 \\
\hline 10 & 18 & 22.96 & 16.30 & 3.79 & 4.25 & 2.84 & 2.02 \\
\hline 0.84 & 1 & 8.92 & 6.34 & 1.43 & 1.61 & 1.07 & 0.76 \\
\hline
\end{tabular}

1 - value calculated for the characteristic length $l=150 \mathrm{~mm}$

2 - value calculated for the characteristic length $l=95 \mathrm{~mm}$

Even though the modification of the test rig brought an intended result, namely the Biot number had a significantly lower value, it still exceeded the value specified in the relevant criterion $(\mathrm{Bi}<0.1)$.

\section{Discussion of results}

The carried out research on RT15 and RT18 materials allowed to plot heating and cooling curves. The curves presented for the cooling process show the measured temperature versus time. In Figs. $8 \mathrm{a}$ and $8 \mathrm{~b}$, the phase transition of the RT18 material, which occurred in the temperature range of $13-20^{\circ} \mathrm{C}$, is clearly visible. As a matter of fact, the phase transition of the RT18 material occurred after a significantly shorter time during the experiment performed on the test rig before its modification. This testifies that the intensive cooling of RT18 before the modification contributed to shortening the duration of the phase transition. After the modification of the test rig, RT18 required more time to change its state. The duration of the phase transition is important when determining the thermophysical parameters of the materials tested. Therefore, both the cooling conditions of samples and the heat exchange process play a significant role. As far as the RT15 material is concerned, both graphs clearly indicate that the phase transition started at a temperature of approx. $20^{\circ} \mathrm{C}$. However, it is hard to say when the change of state was complete. Perhaps RT15 should be cooled by using a temperature lower than $0{ }^{\circ} \mathrm{C}$, which is the cause of the lack of a clear indication of the end of the phase transition. The cooling of RT15 lasted longer than the cooling of RT18 and water and both PCMs finally solidified completely.

During the heating process (Fig. 9), water was the first to reach the ambient temperature. Although the RT15 material needed the longest charging (cooling) time in the ice water, it reached the ambient temperature much faster than the RT18 material. It is also worth noting that at the beginning of the heating process, the temperature of the RT18 material increased faster than the temperature of the water. Once the RT18 reached a temperature of $13.5^{\circ} \mathrm{C}$, the phase transition process began.
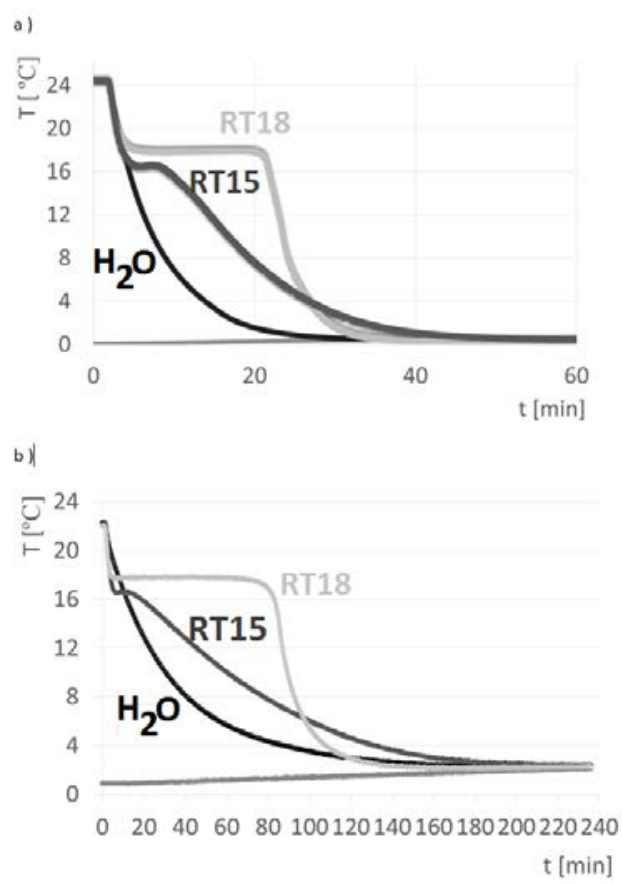

Fig. 8. Cooling curves of the test samples before (a) and after (b) the modification of the test rig.
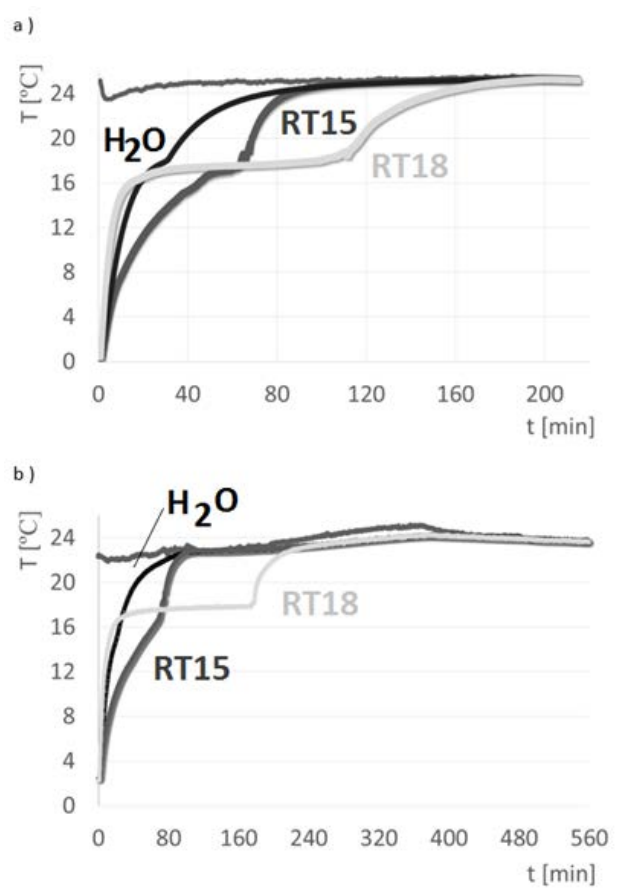

Fig. 9. Heating curves of the test samples before (a) and after (b) the modification of the test rig. 
The value of the Biot number exceeded the value specified in the criterion $(\mathrm{Bi}<0.1)$ both before and after the modification of the test rig. In this case, the assumed criterion was not met. Thus, the T-history method should not be used. The Biot number criterion $(\mathrm{Bi}<0.1)$ assumes that there is an even temperature distribution in a sample. Exceeding a value of 0.1 causes a disruption of this distribution. For this reason, the results concerning the estimation of the latent heat during the cooling process are not reliable and thus the T-history method should not be used for their. However, for the purposes of this article, it was decided to estimate the specific heat of selected PCMs in the ranges in which they occur in the liquid and solid states as well as the generated latent heat on the basis of the T-history method. Calculation of derivatives of the temperature curves of the tested materials yielded two peaks for the RT18 material (at temperatures of $20^{\circ} \mathrm{C}$ and $13.5^{\circ} \mathrm{C}$ ), as shown in Fig. 10 , and one peak for the RT15 material (at a temperature of $20.3{ }^{\circ} \mathrm{C}$ ), as shown in Fig. 11.

According to the assumptions, the obtained temperature peaks were assumed as moments of the start and end of the phase transformation. Thus, the phase transition of the RT18 material occurred (according to the experimental results) in the range of $13.5^{\circ} \mathrm{C}-20{ }^{\circ} \mathrm{C}$. For the RT15 material, it can be only determined that the temperature at the beginning of the phase transition was $20.3^{\circ} \mathrm{C}$. However, it is not possible to ascertain when this transition ended, which makes it difficult to determine the latent heat. The phase change range for RT18 obtained as a result of the experiment differs from the range given by the manufacturer.

Table 3. Comparison of the research results obtained for RT15 and RT18 materials during the cooling process with the manufacturer's data - Rubitherm GmbH.

\begin{tabular}{|c|c|c|c|c|c|}
\cline { 3 - 6 } \multicolumn{2}{c|}{} & \multicolumn{2}{c|}{ RT15 } & \multicolumn{2}{c|}{ RT18 } \\
\cline { 3 - 6 } \multicolumn{2}{c|}{} & 1 & 2 & 1 & 2 \\
\hline $\mathbf{c}_{\mathbf{p}, \mathrm{ml}}$ & $\mathrm{kJ} / \mathrm{kgK}$ & 5.68 & 2 & 5.68 & 2 \\
\hline $\mathbf{c}_{\mathrm{p}, \mathrm{ms}}$ & $\mathrm{kJ} / \mathrm{kgK}$ & 3.69 & 2 & 2.54 & 2 \\
\hline $\mathbf{H}_{\mathbf{m}}$ & $\mathrm{kJ} / \mathrm{kg}$ & 93.2 & 155 & 264.32 & 260 \\
\hline $\mathbf{T}_{\mathbf{1}}$ & ${ }^{\circ} \mathrm{C}$ & 20.3 & 17 & 20 & 19 \\
\hline $\mathbf{T}_{\mathbf{s}}$ & ${ }^{\circ} \mathrm{C}$ & 13.7 & 10 & 13.5 & 17 \\
\hline $\mathbf{T}_{\mathbf{0}}$ & ${ }^{\circ} \mathrm{C}$ & 22.2 & 22 & 22.2 & 26 \\
\hline $\mathbf{T}_{\mathbf{e}}$ & ${ }^{\circ} \mathrm{C}$ & 0.9 & 7 & 0.9 & 11 \\
\hline $\mathbf{T}_{\mathbf{a}}$ & ${ }^{\circ} \mathrm{C}$ & 0.9 & - & 0.9 & - \\
\hline $\begin{array}{c}\text { Temperature } \\
\text { range of } \\
\text { phase change }\end{array}$ & ${ }^{\circ} \mathrm{C}$ & $13.7-$ & $10-17$ & $13.5-20$ & $17-19$ \\
\hline $\begin{array}{c}\text { Range of } \\
\text { measurement } \\
\text { error }\end{array}$ & $\mathrm{kJ} / \mathrm{kg}$ & - & \pm 11.6 & - & \pm 19.5 \\
\hline
\end{tabular}

$\mathbf{1}$ - the result of the experiment

$\mathbf{2}$ - the result provided by the manufacturer

The temperature range in which the test was carried out and the fact that the criterion of the Biot number $(\mathrm{Bi}<0.1)$ was not met both have an impact on the obtained results. The manufacturer tested materials in the temperature range of $11^{\circ} \mathrm{C}-26^{\circ} \mathrm{C}$ and the temperature range in the experiment described herein was $0.8^{\circ} \mathrm{C}-$
$22.3{ }^{\circ} \mathrm{C}$ (which is wider by $6.5^{\circ} \mathrm{C}$ ). For the temperature range of $11^{\circ} \mathrm{C}-26^{\circ} \mathrm{C}$, the latent heat of RT 18 material given by its manufacturer is $268 \mathrm{~kJ} / \mathrm{kg}$. On the other hand, the latent heat of the same material obtained in the experiment for the temperature range of $0.8{ }^{\circ} \mathrm{C}-22.2{ }^{\circ} \mathrm{C}$ is $264.3 \mathrm{~kJ} / \mathrm{kg}$. Considering that the temperature range in which the research was carried out in the experiment is wider than the temperature range of the manufacturer's research, the latent heat calculated on the basis of the experiment would hypothetically have a higher value than if it was calculated for the temperature range that is given by the manufacturer.
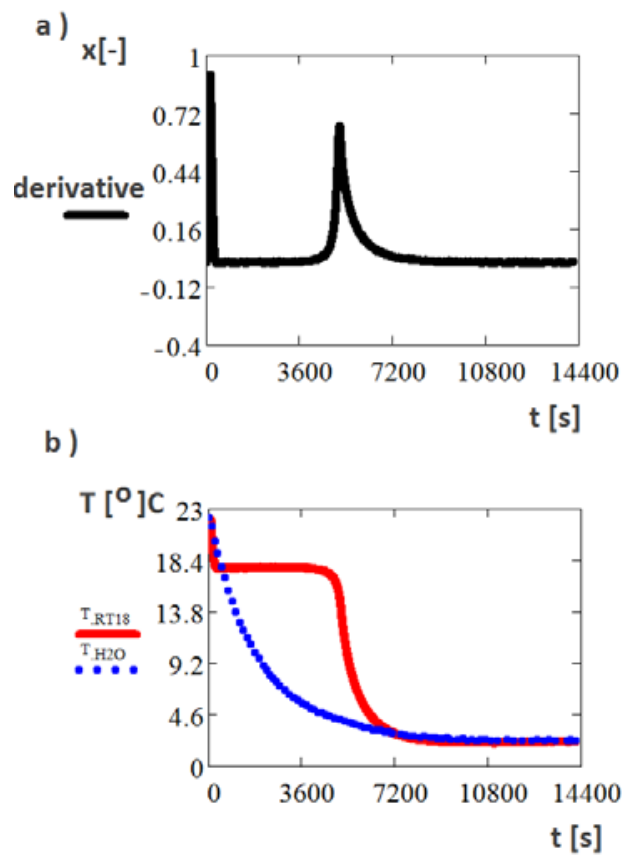

Fig. 10. Derivative of the RT18 temperature vs. time (a) and cooling curves of RT18 and water (b).
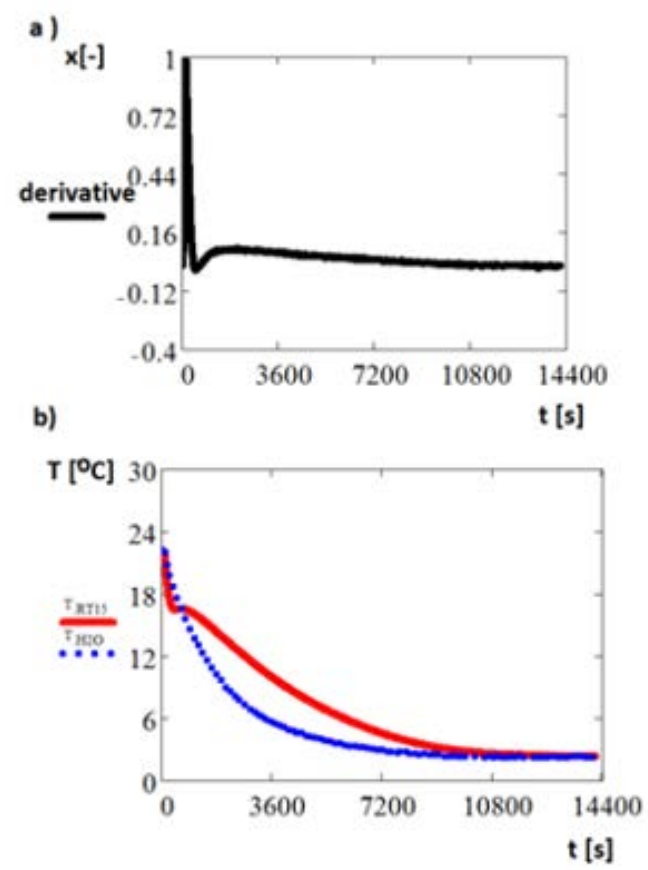

Fig. 11. Derivative of the RT15 temperature vs. time (a) and cooling curves of RT15 and water (b). 
When analysing the results obtained, it should be borne in mind that they are vitiated by an error resulting from exceeding the value of the criterion $(\mathrm{Bi}<0.1)$. Is the error in the estimation of the amount of the latent heat (resulting from the fact that the criterion $\mathrm{Bi}<0.1$ was not met) falls within the range of the manufacturer's measurement error? At this stage, it is very difficult to determine.

\section{Conclusions}

The described T-history method is used to estimate the latent heat and specific heat of a PCM in the liquid and solid state. The use of its modified version for the purposes of the experiment allowed to investigate two PCMs that are available on the market (i.e. RT15 and RT18). These materials were tested as samples with a capacity of $12 \mathrm{ml}$ each (which corresponds to a mass of about $9.2 \mathrm{~g}$ ). The research carried out on large samples of PCMs allows determining the latent heat and specific heat of those materials in a given state of aggregation, and thus the parameters necessary for designing heat and cold storage systems.

The experimental research was performed on RT15 and RT18 materials. The temperatures at which the phase transformation began and ended were clearly identified for the RT18 material. However, the research failed to determine the moment at which the phase transformation was over for the RT15 material. Therefore, it should be investigated whether this moment can be observed if the temperature before commencing the heating process would be lower than $0{ }^{\circ} \mathrm{C}$.

Moreover, it was noticed that the characteristic of the RT15 material given by the manufacturer does not cover temperatures lower than $7^{\circ} \mathrm{C}$. It can, therefore, be assumed that the phase change process covers a wider temperature range than that presented by the manufacturer. As a matter of fact, the conducted research can help in acquiring valuable information regarding the determination of the thermal capacity of heat and cold storage systems in which the RT15 material is used.

A comparison of the manufacturer's data regarding the latent heat with the results obtained from the experiment is possible only for the same temperature range. For this reason, it is planned to determine the enthalpy-temperature relation that will enable to perform such a comparison.

The prerequisite for the use of the T-history method was discussed (i.e. the Biot number criterion has to be fulfilled, $\mathrm{Bi}<0.1$ ), which is of the utmost importance when calculations are carried out. Therefore, before calculating the latent heat, one has to make sure that the criterion is met. Otherwise, the latent heat will be vitiated by a large error.

The preliminary research carried out allowed to determine the scope of further actions aimed at improving the accuracy and reliability of the measurement methodused. At a further stage of the research, the following activities are planned:

- conducting tests using smaller test tubes that will allow for the fulfilment of the Biot number criterion $(\mathrm{Bi}<0.1)$ in the whole temperature range,

- determining the enthalpy-temperature relation for the examined materials,

- applying the method proposed in publication [7], which will allow obtaining more precise results of the latent heat in cases where the Biot number criterion will not be met (in other words, in cases where the Biot number will be greater than 0.1 ).

\section{References}

1. Z. Yinping, J. Yi, A simple method, the T-history method, of determining the heat of Ffusion, specific heat and thermal conductivity of phase- change materials, Measurement Science and Technology, 10, 201-205 (1999)

2. H. Hong, S.K. Kim, Accuracy improvement of Thistory method for measuring heat of fusion of various materials, International Journal of Refrigeration, 27, 360-366 (2004)

3. E.D. Kravvaritis, K.A. Antonopoulos, C. Tzivanidis, Improvements to the measurement of the thermal properties of phase change materials, Measurement Science and Technology, 21, 9 (2010)

4. H. Mehling, H.P. Ebert, P. Schossig, Development of standards for materials testing and quality control of PCM, $7^{\text {th }}$ IIR Conference on Phase Change Materials and Slurries for Refrigeration and Air Conditioning, (2006)

5. W. Pudlik, Wymiana $i$ wymienniki ciepta, Wydanie V - cyfrowe, Wydawnictwo Politechniki Gdańskiej, Gdańsk (2012), published in "Pomorska Biblioteka Cyfrowa"(20.05.2018):http://pbc.gda.pl/Content/440 4/wymiana-i-wymienniki-final.pdf

6. A. Solé, L. Miró, C. Barreneche, I. Martorell, L.F. Cabeza, Review of the T-history method to determine thermophysical properties of phase change materials (PCM), Renewable and Sustainable Energy Reviews, 26, 425-436 (2013)

7. B. Delcroix, M. Kummert, A. Daoud, J. Bouchard, Influence of experimental conditions on measured thermal properties used to model phase change materials, Building Simulation, 8, 637-650 (2015)

8. https://www.rubitherm.eu/en/index.php/productcate gory/organische-pcm-rt

Presented work was supported by SuPREME project. This project has received funding from the European Union's Horizon 2020 research and innovation programme under grant agreement No. 692197. 